\title{
ZNF24 is upregulated in prostate cancer and facilitates the epithelial-to-mesenchymal transition through the regulation of Twist1
}

\author{
XIANGJIANG HUANG ${ }^{1,2}$, NANXIN LIU $^{1,2}$ and XING XIONG ${ }^{1,2}$ \\ ${ }^{1}$ Department of Urology Surgery, Shenzhen People's Hospital; ${ }^{2}$ Department of Urology Surgery, \\ Second Clinical Medical College of Jinan University, Shenzhen, Guangdong 518020, P.R. China
}

Received February 26, 2019; Accepted December 12, 2019

DOI: 10.3892/ol.2020.11456

\begin{abstract}
Zinc finger protein 24 (ZNF24) has been demonstrated to regulate proliferation, differentiation and migration as well as invasion in several types of cells. However, the molecular role and clinical effects of ZNF24 in prostate cancer (PCa) remain unclear. The present study revealed that ZNF24 expression is upregulated in $\mathrm{PCa}$, and associated with tumor volume, Gleason score, pathological grade and metastasis. Wound healing and Transwell invasion assays revealed that ectopic ZNF24 expression facilitated cell migration and invasion through the Twist1-induced epithelial-to-mesenchymal transition (EMT) process. In addition, colony formation and Cell Counting Kit-8 assays were used to determine the regulatory effects of ZNF24 on proliferation. The results suggested that ZNF24 also promoted cell proliferation in PCa. ZNF24 acted as an oncogene and promoted migration, invasion and EMT of PCa cells via the regulation of Twist1.
\end{abstract}

\section{Introduction}

Prostate cancer (PCa) has become the most frequently diagnosed cancer in the male genitourinary system in the United States, in 2013 (1). The main methods for the early diagnosis of $\mathrm{PCa}$ include transrectal ultrasound, $\mathrm{PCa}$-specific antigen determination and digital rectal examination $(2,3)$. In addition, increasing evidence has indicated that inflammation serves a key role in the pathogenesis of PCa via regulation of the tumor microenvironment by releasing growth factors and proinflammatory cytokines, and, therefore, influencing tumor development (4-6). Therefore, an improved understanding of the detailed molecular mechanism underlying metastasis is

Correspondence to: Dr Xiangjiang Huang, Department of Urology Surgery, Shenzhen People's Hospital, 1017 Dongmen North Road, Shenzhen, Guangdong 518020, P.R. China

E-mail: huangxjjiang@163.com

Key words: zinc finger protein 24, invasion, epithelial-tomesenchymal transition, Twist1, prostate cancer urgently required in order to develop therapies and prevent PCa.

Zinc finger protein 24 (ZNF24; also known as KOX17, Zfp191 or ZNF191) is a member of the Kruppel-like zinc finger transcription factor family (7). Its $\mathrm{N}$ terminus harbors a SCAN domain that primarily functions as a dimerization domain in zinc finger proteins (8), and its $\mathrm{C}$ terminus contains four $\mathrm{C} 2 \mathrm{H} 2$ zinc finger motifs that serve as DNA binding domains (9). ZNF24 is ubiquitously expressed during embryonic development and in adult tissues $(10,11)$, suggesting that it serves a key role in multiple different cell types. Independent studies have demonstrated that knockout of ZNF24 results in premature death at different time points during development $(12,13)$, suggesting that ZNF24 serves a crucial role in regulating organ development. In addition, $\mathrm{ZNF} 24$ has been revealed to regulate proliferation, differentiation, migration and invasion in several different types of cancer, such as hepatocellular carcinoma and gastric cancer $(14,15)$. Upregulation of ZNF24 in neural progenitor cells maintains these cells in an actively proliferating state and suppresses neuronal differentiation (16). Furthermore, the role of ZNF24 in regulating cell migration and invasion has been investigated in aortic vascular smooth muscle cells, where ZNF24 promotes cell migration (17). However, to the best of our knowledge, the molecular role and clinical effects of ZNF24 in PCa remain unclear.

The epithelial-to-mesenchymal transition (EMT) is the initial step of migration (18). Loss of epithelial cell-cell adhesion and the gain of mesenchymal-like morphology are the main characteristics of the EMT $(19,20)$. EMT can enhance the invasive ability of tumor cells, and enable them to leave their primary sites, which leads to metastasis (21). Several studies have revealed that EMT could be activated by EMT-related transcription factors, including Twist, Slug and Snail $(22,23)$.

The present study evaluated the gene and protein expression levels of ZNF24 in PCa tissue samples and cancer cell lines. Subsequently, gain and loss of function assays were performed to specifically up- or downregulate ZNF24 in PCa cell lines (PC-3 and DU-145) to assess its effect on PCa migration and invasion, as well as growth. Furthermore, the present study examined whether Twist1 was a downstream target of ZNF24. The results revealed a novel mechanism of ZNF24 in PCa migration and invasion. 


\section{Materials and methods}

Patient tissue specimens. A total of 68 human PCa (mean, 65.3 years; range, 24-74 years) and 43 matched adjacent non-tumor tissues were obtained from patients who underwent surgical resection at Shenzhen People's Hospital (Shenzhen, China) between May 2008 and December 2012 for reverse transcription-quantitative PCR (RT-qPCR) and western blotting analyses. None of the patients had undergone chemotherapy or radiotherapy prior to surgery. Gleason score and pathological stage of prostate patients were determined as described previously $(24,25)$. All tissues were immediately frozen in liquid nitrogen and stored at $-80^{\circ} \mathrm{C}$. The present study was approved by the Clinical Research Ethics Committee of Shenzhen People's Hospital. All patients provided written informed consent prior to experiments.

Cell culture. The human PCa cell lines PC-3 and DU-145, and the human normal prostate cell line RWPE-1 were purchased from The Cell Bank of Type Culture Collection of Chinese Academy of Sciences and cultured in RPMI-1640 medium (Thermo Fisher Scientific, Inc.) supplemented with $10 \%$ fetal bovine serum (FBS; Thermo Fisher Scientific, Inc.), $100 \mathrm{U} / \mathrm{ml}$ penicillin and $100 \mathrm{mg} / \mathrm{ml}$ streptomycin in a humidified atmosphere at $37^{\circ} \mathrm{C}$ with $5 \% \mathrm{CO}_{2}$.

Transfection. ZNF24 short hairpin RNA (shRNA) plasmid was purchased from Hanbio Biotechnology Co., Ltd. The sequences were as follows: ZNF24 shRNA, 5'-CCGGGA GGATTTGGAGAGTGAACTTCTCGAGAAGTTCACTCT CCAAATCCTCTTTTTG-3'; and control shRNA (shControl), 5'-CCGGGCTGACCCTGAAGTTCATCCTCGAGGATGAA CTTCAGGGTCAGCTTTTTG-3'. The pcDNA3.1 (vector) and pcDNA-3.1-ZNF24 plasmid were purchased from Vigene Biosciences. The plasmids $(2.5 \mu \mathrm{g})$ were transfected using Lipofectamine $^{\circledR} 2000$ (Thermo Fisher Scientific, Inc.) according to the manufacturer's protocol. Following transfection for $48 \mathrm{~h}$, cells were collected and prepared for further experiments.

Western blotting. Tissue samples and cells were lysed using RIPA buffer (Beyotime Institute of Biotechnology). The protein concentration was measured using a BCA kit (Pierce; Thermo Fisher Scientific, Inc.) according to the manufacturer's protocol. Protein samples $(\sim 40 \mu \mathrm{g})$ were separated via SDS-PAGE (10\% gel) and the protein was then transferred onto a PVDF membrane, followed by incubation with $5 \%$ skimmed milk in TBS with $0.1 \%$ Tween-20 at $4^{\circ} \mathrm{C}$ overnight. Subsequently, the membranes were incubated with primary antibodies at $4^{\circ} \mathrm{C}$ overnight. The primary antibodies were: ZNF24 (1:2,000 dilution; cat. no. ab176589; Abcam), E-cadherin (1:1,000 dilution; cat. no. 3195; Cell Signaling Technology, Inc.), N-cadherin (1:1,000 dilution; cat. no. 13116; Cell Signaling Technology, Inc.) and $\beta$-actin (1:5,000 dilution; cat. no. ab179467; Abcam). Following three washes with TBST at room temperature for $5 \mathrm{~min}$, membranes were incubated in with secondary horseradish peroxidase-conjugated goat anti-rabbit antibody (1:5,000 dilution; cat. no. ab205718; Abcam) for $1 \mathrm{~h}$ at room temperature. Following three washes with TBST at room temperature for $5 \mathrm{~min}$, the blots were visualized using an ECL solution kit (Pierce; Thermo Fisher Scientific, Inc.) according to the manufacturer's protocol. Each experiment was repeated independently three times.

$R T-q P C R$. RNA was harvested from tissue specimens and cells using TRIzol ${ }^{\circledR}$ reagent (Thermo Fisher Scientific, Inc.) according to the manufacturer's protocol. RNA $(2 \mu \mathrm{g})$ was used to synthesize cDNA using the High-Capacity cDNA Reverse Transcription kit (Thermo Fisher Scientific, Inc.) according to the manufacturer's protocol. The reaction conditions used were as follows: $42^{\circ} \mathrm{C}$ for $60 \mathrm{~min}$ and $70^{\circ} \mathrm{C}$ for $5 \mathrm{~min}$. Subsequently, SYBR Green Master (Roche Diagnostics $\mathrm{GmbH}$ ) was utilized for the qPCR using the ABI PRISM 7500 system (Applied Biosystems; Thermo Fisher Scientific, Inc.). The reaction conditions used were as follows: Pre-denaturation at $95^{\circ} \mathrm{C}$ for $4 \mathrm{~min}, 30$ cycles of denaturation at $94^{\circ} \mathrm{C}$ for $30 \mathrm{sec}$, annealing at $59^{\circ} \mathrm{C}$ for $30 \mathrm{sec}$ and extension at $72^{\circ} \mathrm{C}$ for $1 \mathrm{~min}$. The thermocycling conditions were as follows: $5 \mathrm{~min}$ at $65^{\circ} \mathrm{C}$, $5 \mathrm{~min}$ at $42^{\circ} \mathrm{C}$, and $1 \mathrm{~min}$ at $85^{\circ} \mathrm{C}$. GAPDH was used as the internal reference for other genes. The $2^{-\Delta \Delta \mathrm{Cq}}$ method was utilized to calculate the relative expression of target genes (26). The primers were as follows: ZNF24: Forward, 5'-GTTCCT GAGCCGGAGGTTT-3', reverse 5'-CTCAGGGCAATACCC CGTTT-3'; E-cadherin: Forward, 5'-AGTGACTGATGC TGATGCCC-3'; reverse, 5'-AATGTACTGCTGCTTGGC CT; N-cadherin: Forward, 5'-GTGCATGAAGGACAGCCT CT-3'; reverse, 5'-TGGAAAGCTTCTCACGGCAT-3'; Twist1: Forward, 5'-TCAAGAGGTCGTGCCAATCA-3'; reverse, 5'-TTGCAGGCCAGTTTGATCCC-3'; Slug: Forward, 5'-GCT ACCCAATGGCCTCTCTC-3'; reverse, 5'-CTTCAATGG CATGGGGGTCT-3'; Snail: Forward, 5'-CGAGTGGTTCTT CTGCGCTA-3'; reverse, 5'-GGGCTGCTGGAAGGTAAA CT-3'; GAPDH: Forward, 5'-CCATGGGGAAGGTGAAGG TC-3'; and reverse, 5'-GCGCCCAATACGACCAAATC-3'. Each experiment was repeated independently three times.

Colony-formation assay. PC-3 and DU-145 cells were placed into 6-well plates at a density of $5 \times 10^{3}$ cells/well. Cells were cultured for 2 weeks with serum-free RPMI-1640 medium. Subsequently, the cells were fixed with $100 \%$ methanol at room temperature for $5 \mathrm{~min}$ and stained with $0.5 \%$ crystal violet at room temperature for $10 \mathrm{~min}$. Each experiment was repeated independently three times.

Wound healing assay. A total of $5 \times 10^{5}$ PC-3 and DU-145 cells were plated in a 6-well plate. When the cell confluence reached $85-100 \%$, a $10-\mu l$ pipette was used to make scratches on the cells covering the bottom of the plate of the same width. The cells were then washed three times with PBS and then cultured with serum-free RPMI-1640 medium. At 0 and $48 \mathrm{~h}$ after scratching, the migration distance in the scratched area was examined, and images were captured under a light microscope (magnification, $\mathrm{x} 40$ ). The relative distance of migration $=($ width at $0 \mathrm{~h}$-width at $48 \mathrm{~h}$ )/width at $48 \mathrm{~h}$. Each experiment was repeated independently three times.

Transwell invasion assay. Invasion assays were performed as previously described by using Matrigel invasion chambers (Thermo Fisher Scientific, Inc.) $(27,28)$. In brief, a total of $2 \times 10^{4}$ PC-3 and DU-145 cells were transfected with ZNF24 or 
ZNF24 shRNA, respectively. After transfection for $48 \mathrm{~h}$, cells were counted and re-suspended in serum-free RPMI-1640 medium. Cells $\left(\sim 4 \times 10^{4}\right)$ were seeded in 24-well Matrigel invasion chambers (Thermo Fisher Scientific, Inc.). The upper chamber was filled with serum-free RPMI-1640 medium and the bottom chamber was filled with RPMI-1640 medium supplemented with $10 \%$ FBS. Cells were incubated at $37^{\circ} \mathrm{C}$ for $20 \mathrm{~h}$. The cells were stained with $0.5 \%$ crystal violet at room temperature for $10 \mathrm{~min}$ and then washed with PBS. The cells on the surface of the chamber were removed using a cotton-tipped swab. The cells that had invaded to the bottom chamber were quantified using a light microscope (magnification, $x 40$ ). Each experiment was repeated independently three times.

Dual luciferase reporter assay. A dual luciferase reporter assay was utilized to further verify the targeting association of ZNF24 and Twist1. Briefly, the promoter region of Twist1 (-2000, +200) was cloned into pGL3-basic plasmid (Vigene Biosciences). A mixture of $1 \mu \mathrm{g}$ pGL3-Twist1, $2 \mu \mathrm{g}$ ZNF24 or ZNF24 shRNA and 20 ng Renilla plasmid was transfected into a total of $5 \times 10^{5} \mathrm{PC}-3$ and DU-145 cells using Lipofectamine ${ }^{\circledR} 2000$ (Thermo Fisher Scientific, Inc.) according to the manufacturer's protocol. Following transfection for $24 \mathrm{~h}$, the cells were collected and lysed. Firefly and Renilla luciferase activities were measured with a Dual-Luciferase Reporter Assay system (Promega Corporation) according to the manufacturer's protocol. Renilla luciferase activities were used as an internal control for transfection efficiency. Each experiment was repeated independently three times.

Chromatin immunoprecipitation (ChIP) and quantitative $(q)$ ChIP assay. ChIP and qChIP analyses were performed using an EZ-ChIP kit (EMD Millipore) according to the manufacturer's protocol. In brief, PC-3 and DU-145 cells were cultured to $80-100 \%$ confluence, and the chromatin was cross-linked by $1 \%$ formaldehyde at $37^{\circ} \mathrm{C}$ for $15 \mathrm{~min}$. Subsequently, cross-linked chromatin was sonicated (1 sec on, 1 sec off; $3 \times 20$ times) at $4^{\circ} \mathrm{C}$ to generate $200-1,000$ bp fragments. Next, $5 \mu \mathrm{g}$ anti-immunoglobulin $\mathrm{G}$ (IgG) antibody (cat. no. ab171870; Abcam) or anti-ZNF24 (cat. no. ab176589; Abcam) were used to immunoprecipitate chromatin fragments at $4^{\circ} \mathrm{C}$ overnight. IgG antibody was used as the control. After purifying the antibody-interact DNA, RT-qPCR was conducted to analyze the precipitated chromatin DNA, as aforementioned. The primer sequences were as follows: Twist1: Forward, 5'-AAGGGA TGGACCTGAAACGG-3'; and reverse, 5'-GGCAAACTG GAAGCAGCAAA-3'. The qPCR conditions were as follows: $5 \mathrm{~min}$ at $98^{\circ} \mathrm{C}$, denaturation at $98^{\circ} \mathrm{C}$ for $30 \mathrm{sec}$, annealing at $56^{\circ} \mathrm{C}$ for $30 \mathrm{sec}$ and extension at $72^{\circ} \mathrm{C}$ for $20 \mathrm{sec}$, performed for 32 cycles.

Cell Counting Kit-8 (CCK-8) assay. Cells $\left(\sim 3 \times 10^{3}\right)$ in $200 \mu 1$ RPMI-1640 medium were placed in 96-well plates. A total of six parallel wells were prepared for each group. CCK-8 solution (20 $\mu \mathrm{l}$; Beyotime Institute of Biotechnology) was added into each well at $0,24,48$ and $72 \mathrm{~h}$, and incubated for $1 \mathrm{~h}$ at $37^{\circ} \mathrm{C}$. The optical density value was measured at $450 \mathrm{~nm}$ in each well. Each experiment was repeated independently three times.
Bioinformation analysis. The expression of ZNF24 in prostate tissue was obtained from The Human Protein Atlas database (https://www.proteinatlas.org). The details are as follows: Antibody number, CAB025642 and HPA024062; male, age 76; Patient ID: 2932.

Statistical analysis. SPSS v19.0 software (IBM Corp.) was employed for data analysis. Values in all graphs are presented as the mean \pm standard deviation. For Table I, the mean value of ZNF24 mRNA content (6.4) in tumor cells was set as the standard. Therefore, higher values than the standard value were defined as high expression, and lower values than the standard value were defined as low expression. The $\chi^{2}$ test was applied for comparisons between ZNF24 expression and clinical information of patients with PCa. Student's t-test was applied for comparisons between two groups, and one-way ANOVA followed by Tukey's post-hoc test was applied for comparisons among multiple groups. $\mathrm{P}<0.05$ was considered to indicate a statistically significant difference.

\section{Results}

ZNF24 is upregulated in PCa tissues and cell lines. To decipher the function of ZNF24 in $\mathrm{PCa}$, the present study first detected ZNF24 expression in 68 human PCa and 43 adjacent non-tumor tissues using RT-qPCR and western blotting analyses. mRNA and protein expression levels of ZNF24 in tumor tissues were markedly higher compared with those in adjacent non-tumor tissues ( $\mathrm{P}<0.05$; Fig. 1A). Additionally, ZNF24 expression in PCa cell lines, including PC-3 and DU-145, was detected, and the human normal prostate RWPE-1 cell line served as the control. Consistent with the results in the tissue samples, the expression levels of ZNF24 in the PCa cell lines were significantly higher than those in RWPE-1 cells ( $\mathrm{P}<0.05$; Fig. 1B). In order to further investigate the function of ZNF24 in $\mathrm{PCa}$, the association between ZNF24 expression and pathological characteristics of patients with PCa was evaluated. ZNF24 expression was positively associated with tumor volume, Gleason score, pathological stage and metastasis (Table I). Additionally, the present study analyzed the expression levels of ZNF24 in prostate tissues using data from The Human Protein Atlas database (https://www.proteinatlas.org; data not shown). Consistent with the aforementioned findings, ZNF24 was upregulated in PCa. These findings suggest that ZNF24 may serve an essential role in $\mathrm{PCa}$.

ZNF24 promotes $P$ Ca cell migration and invasion in vitro. In order to further investigate the role of ZNF24 overexpression in carcinogenesis and progression of $\mathrm{PCa}$, the present study induced overexpression or knockdown of ZNF24 in PC-3 and DU-145 cells. The transfection efficiency of ZNF24 was confirmed by western blotting and RT-qPCR analyses $(\mathrm{P}<0.05$; Fig. 2A). Subsequently, wound healing and Transwell invasion assays were performed to determine the effects of ZNF24 on cell migration and invasion in $\mathrm{PCa}$. The results of the wound healing assay indicated that overexpression of ZNF24 markedly promoted the relative migratory distance compared with that in the vector group; however, inhibition of ZNF24 resulted 
Table I. Clinicopathological variables of 68 patients with prostate cancer.

ZNF24 expression

\begin{tabular}{|c|c|c|c|c|}
\hline Variables & Number, $\mathrm{n}(\%)(\mathrm{n}=68)$ & Low, n (\%) $(\mathrm{n}=26)$ & High, $n(\%)(n=42)$ & P-value \\
\hline \multicolumn{5}{|l|}{ Age, years } \\
\hline$<66$ & $30(44.1)$ & $13(50.0)$ & $17(40.5)$ & 0.442 \\
\hline$\geq 66$ & $38(55.9)$ & $13(50.0)$ & $25(59.5)$ & \\
\hline \multicolumn{5}{|c|}{ Tumor volume, $\%$} \\
\hline$<30$ & $26(38.2)$ & $14(53.8)$ & $12(28.6)$ & 0.037 \\
\hline$\geq 30$ & $42(61.8)$ & $12(46.2)$ & $30(71.4)$ & \\
\hline \multicolumn{5}{|c|}{ Gleason score (24) } \\
\hline$<6$ & $32(47.1)$ & $17(65.4)$ & $15(35.7)$ & 0.017 \\
\hline$>7$ & $36(52.9)$ & $9(34.6)$ & $27(64.3)$ & \\
\hline \multicolumn{5}{|c|}{ Pathological stage (25) } \\
\hline $\mathrm{I}-\mathrm{II}$ & $31(45.6)$ & $16(61.5)$ & $15(35.7)$ & 0.038 \\
\hline III-IV & $37(54.4)$ & $10(38.5)$ & $27(64.3)$ & \\
\hline \multicolumn{5}{|l|}{ Metastasis } \\
\hline Yes & $29(42.6)$ & 7 (26.9) & $22(52.4)$ & 0.039 \\
\hline No & $39(57.4)$ & $19(70.1)$ & $20(47.6)$ & \\
\hline
\end{tabular}

ZNF24 expression in prostate cancer tissues from patients was analyzed by reverse transcription-quantitative PCR. The association between ZNF24 expression and each clinicopathological variable was analyzed using the $\chi^{2}$ test. ZNF24, zinc finger protein 24 .
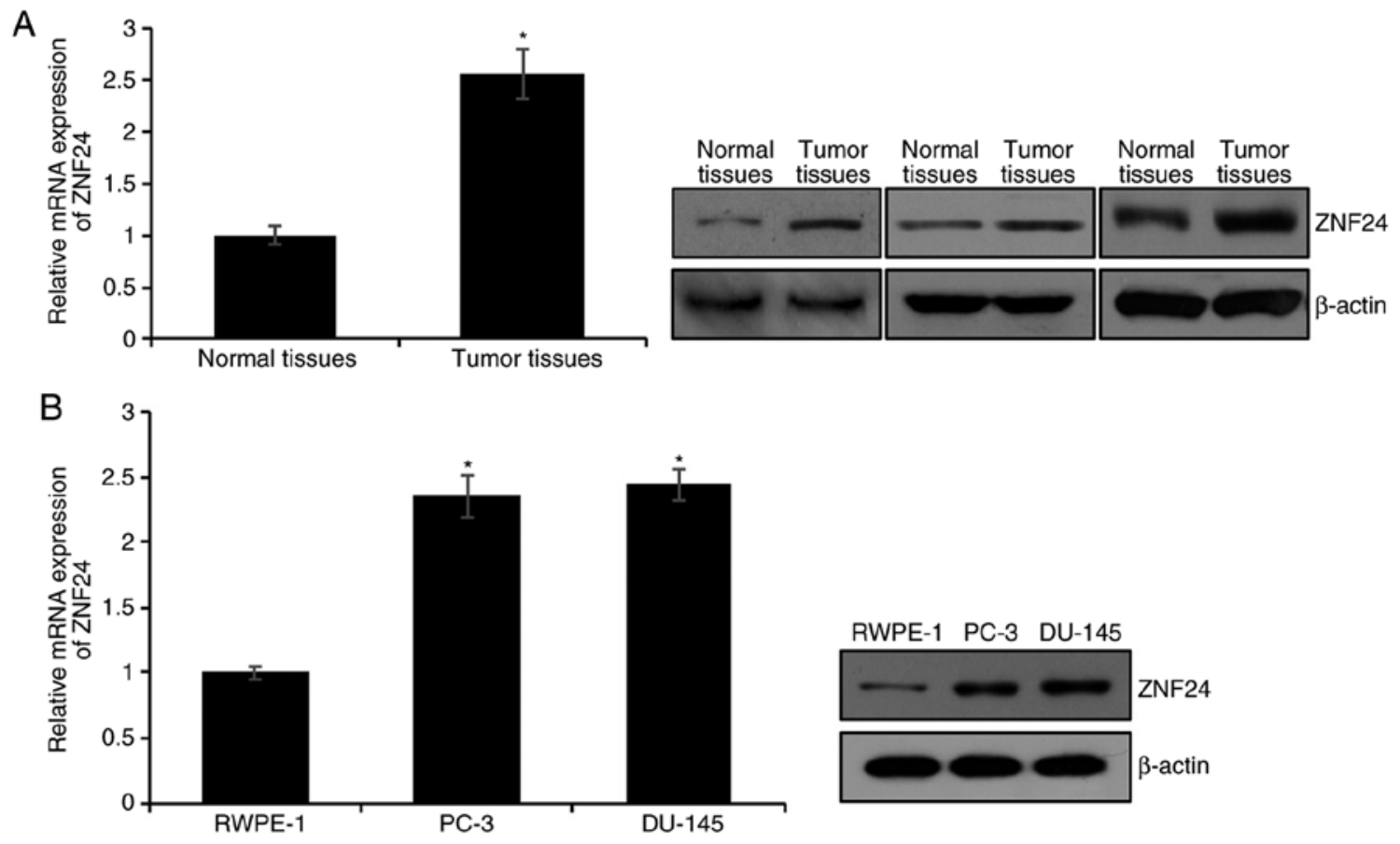

Figure 1. ZNF24 is upregulated in PCa tissues and cell lines. (A) Expression levels of ZNF24 in PCa and adjacent normal samples were determined using RT-qPCR and western blotting assays. "P $<0.05$ vs. normal tissues. (B) RT-qPCR and western blotting assays were performed to compare the expression levels of ZNF24 in PCa cell lines (PC-3 and DU-145) with the expression levels of ZNF24 in the control cell line (RWPE-1). "P<0.05, PC-3 or DU-145 vs. RWPE-1. PCa, prostate cancer; RT-qPCR, reverse transcription-quantitative PCR; ZNF24, zinc finger protein 24.

in the opposite result, and the relative migratory distance was lower than that in shControl group ( $\mathrm{P}<0.05$; Fig. 2B). Additionally, similar results were observed in Transwell invasion assays. The numbers of invasive cells were increased when cells overexpressed ZNF24, and decreased when ZNF24 was knocked-down $(\mathrm{P}<0.05$; Fig. 2C). Overall, the results demonstrated that ZNF24 promoted $\mathrm{PCa}$ cell migration and invasion in vitro. 
A
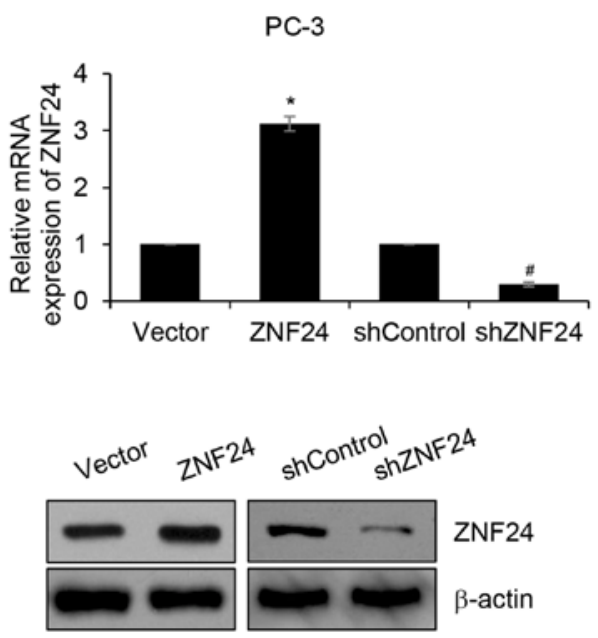

B
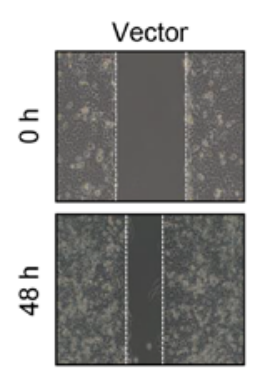

PC-3

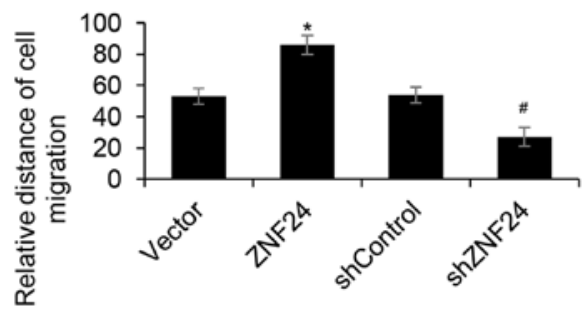

C

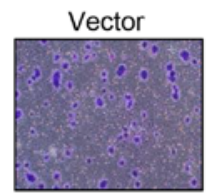

PC-3

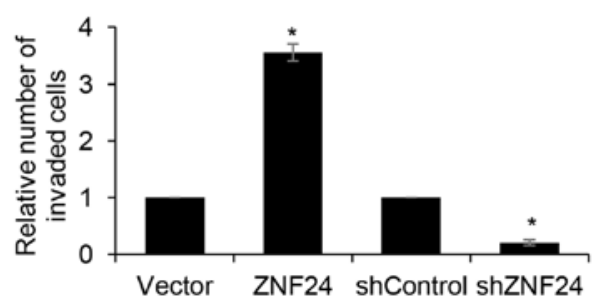

DU-145
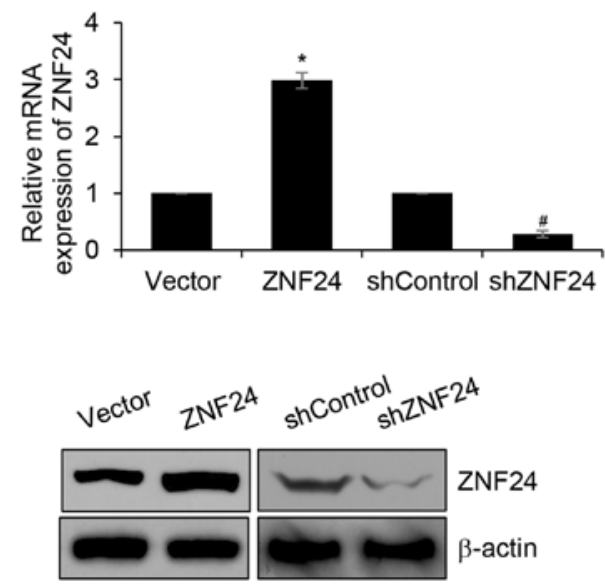

shZNF24
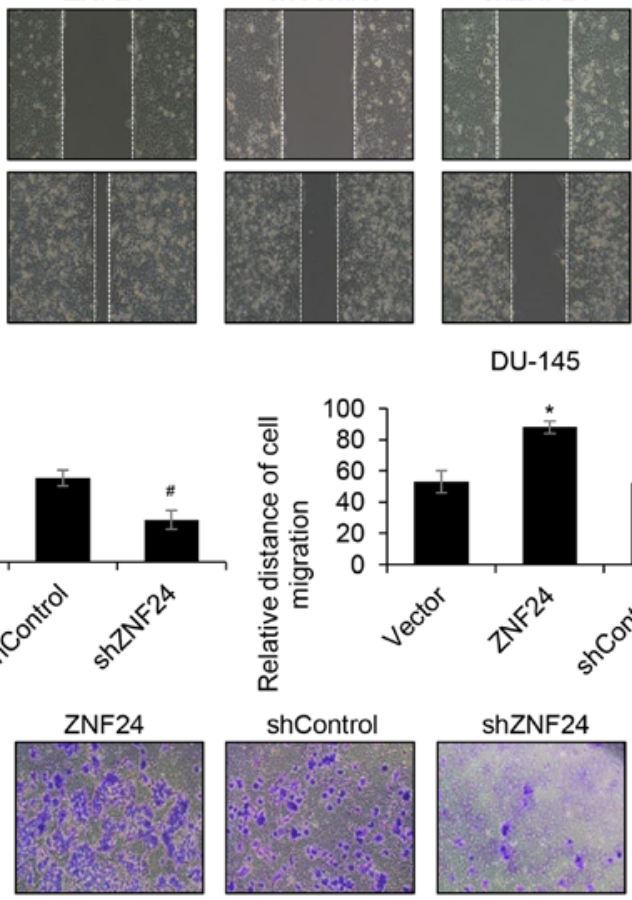

DU-145

ZNF24
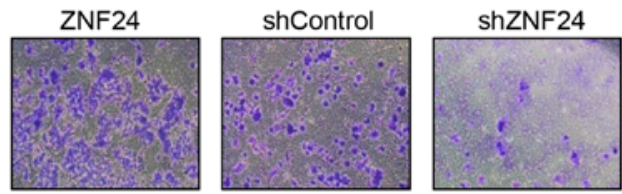

DU-145

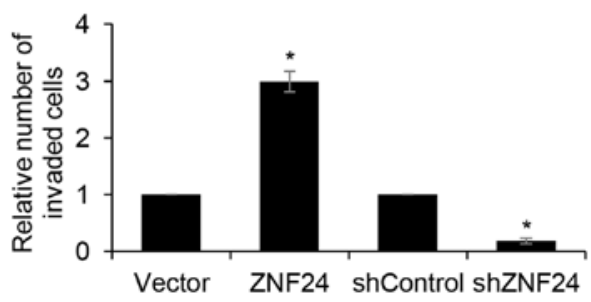

Figure 2. ZNF24 promotes prostate cancer cell migration and invasion in vitro. ZNF24 was overexpressed or knocked-down in PC-3 and DU-145 cells. (A) ZNF24 expression was assessed using RT-qPCR and western blotting assays. Representative images of PC-3 and DU-145 cells in (B) wound healing assay was performed to determine the effect of ZNF24 on cell migration. Magnification, $\mathrm{x} 40$. (C) Transwell invasion assays assay was performed to determine the effect of ZNF24 on cell invasion. Magnification, $\mathrm{x} 40$. Bar graphs display the statistics for cell counts. ${ }^{*} \mathrm{P}<0.05$, ZNF24 vs. vector; ${ }^{\#} \mathrm{P}<0.05$, shZNF24 vs. shControl. sh, short hairpin RNA; ZNF24, zinc finger protein 24.

ZNF24 facilitates PCa cell invasion and metastasis through regulation of EMT.In order to further investigate the underlying mechanism of enhanced migration and invasion by ZNF24 in PCa, the effect of ZNF24 on EMT was investigated. The typical EMT phenotype is downregulation of E-cadherin and upregulation of $\mathrm{N}$-cadherin (29). The present study revealed that overexpression of ZNF24 resulted in elevated expression of $\mathrm{N}$-cadherin and decreased expression of E-cadherin $(\mathrm{P}<0.05$; Fig. 3A). Conversely, knockdown of ZNF24 led to the opposite result, $\mathrm{N}$-cadherin expression was decreased, and E-cadherin 
A
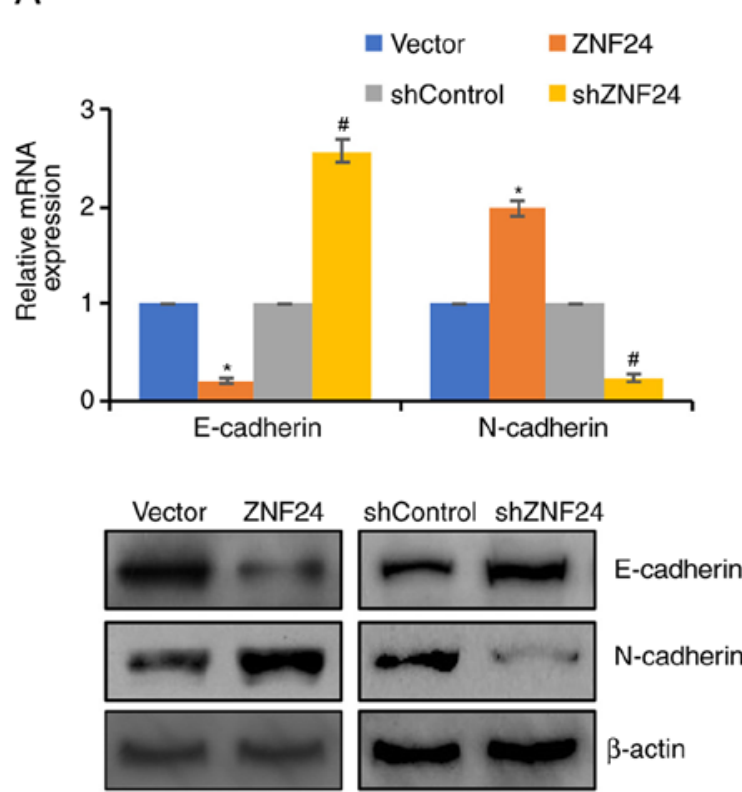

B

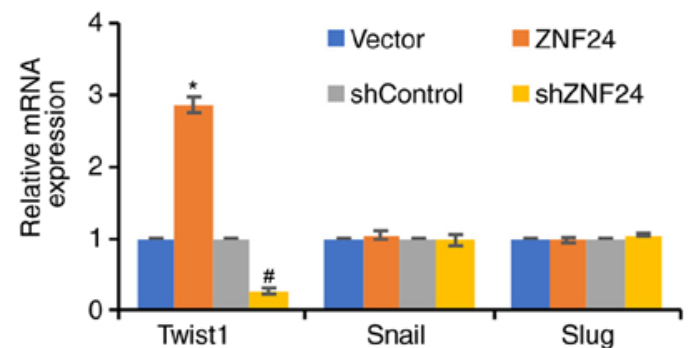

Vector ZNF24 shControl shZNF24

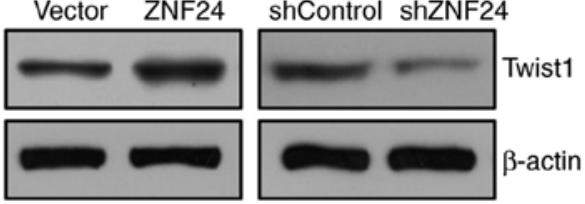

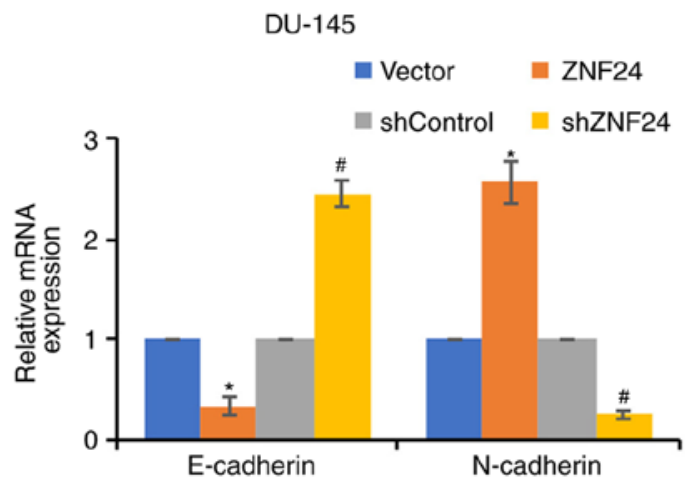

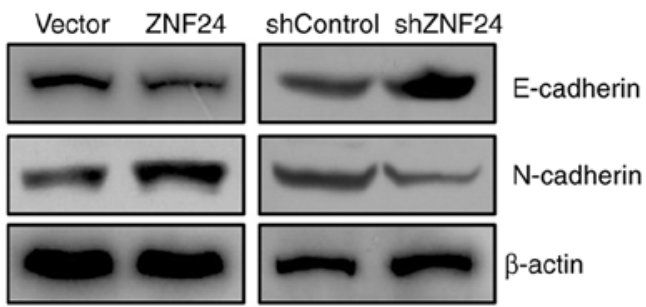

DU-145

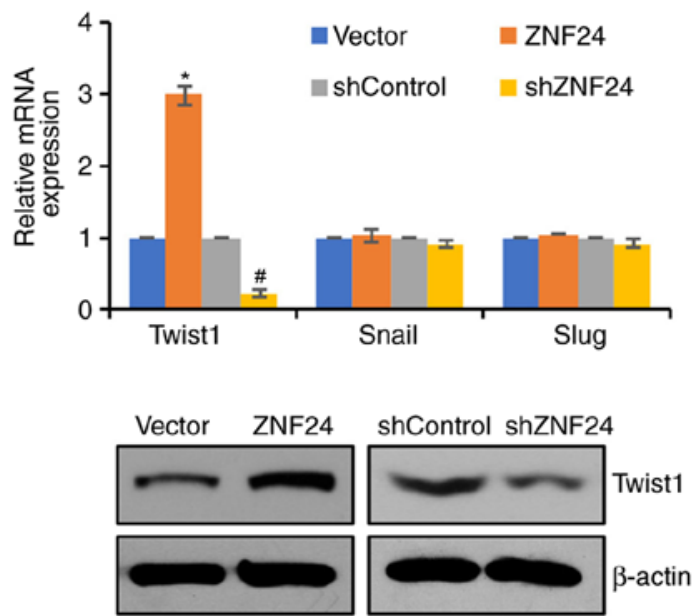

Figure 3. ZNF24 facilitates PCa cell invasion and metastasis through regulation of EMT. ZNF24 was overexpressed or knocked-down in PC-3 and DU-145 cells. (A) Expression levels of EMT-markers, including E-cadherin and N-cadherin, were identified by RT-qPCR and western blotting assays. (B) Twist1 expression was detected by RT-qPCR and western blotting assays. ${ }^{*} \mathrm{P}<0.05$, ZNF24 vs. vector; ${ }^{*} \mathrm{P}<0.05$, shZNF24 vs. shControl. EMT, epithelial-mesenchymal transition; PCa, prostate cancer; RT-qPCR, reverse transcription-quantitative PCR; sh, short hairpin RNA; ZNF24, zinc finger protein 24.

expression was increased $(\mathrm{P}<0.05$; Fig. 3A). Furthermore, based on the analysis of ZNF24 expression in the prostate adenocarcinoma database (Fred Hutchinson CRC, Nat Med 2016) (30), it was identified that ZNF24 was upregulated in PCa. Additionally, the EMT-associated transcription factor, Twist1, was upregulated in PCA. However, ZNF24 expression had no effect on other EMT-associated transcription factors, including Slug and Snail (Fig. 3B). Therefore, ZNF24 may promote EMT through regulation of Twist1 in PCa. These results suggest that ZNF24 facilitated $\mathrm{PCa}$ cell invasion and metastasis through regulation of EMT.

Twistl is a target gene of ZNF24. In order to ascertain whether ZNF24 could directly regulate Twist1 expression, ChIP and qChIP assays, and a dual luciferase activity assay were performed. The fold of enrichment of Twistl was increased almost 7-fold when ZNF24 was overexpressed, suggesting that ZNF24 could bind the promoter region of Twist1 $(\mathrm{P}<0.05$; Fig. 4A). Subsequently, a dual luciferase reporter assay was performed. The results demonstrated that the luciferase activity was significantly increased when ZNF24 was overexpressed, compared with the vector group. Conversely, shZNF24 transfection resulted in decreased luciferase activity, indicating that ZNF24 transcriptionally activated Twist1 expression $(\mathrm{P}<0.05$; Fig. 4B). The aforementioned results suggest that Twist1 is a target gene of ZNF24.

Upregulation of ZNF24 promotes $P C$ a cell proliferation. Since ZNF24 expression was associated with tumor volume, it was assumed that ZNF24 may serve a role in cell proliferation. In order to verify this hypothesis, in vitro experiments were used to examine the effects of ZNF24 on cell proliferation. A CCK-8 assay was used to detect cell proliferation at $0,24,48$ and $72 \mathrm{~h}$. The cell proliferation rate was markedly increased 
A

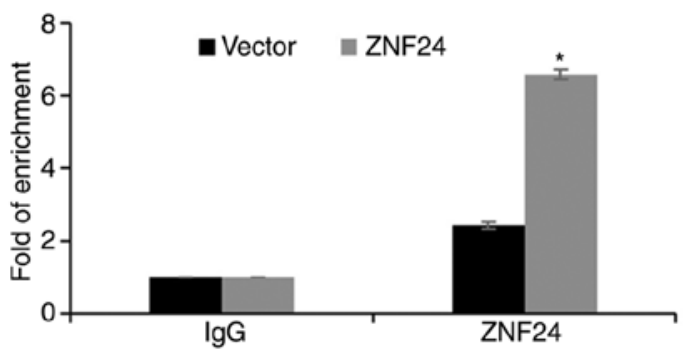

B

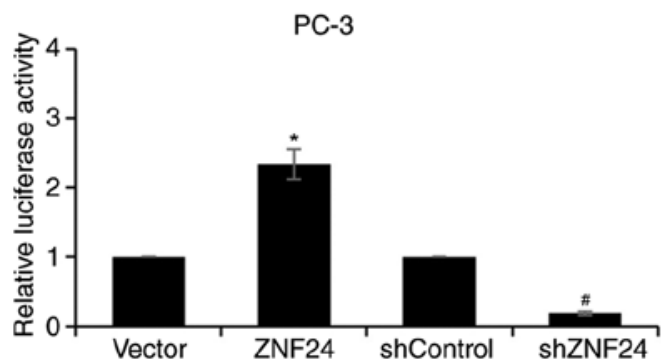

DU-145

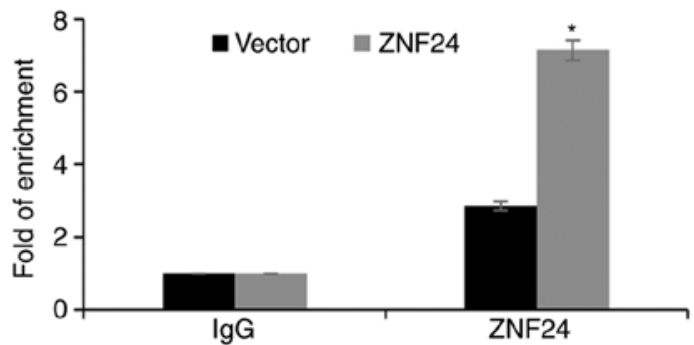

DU-145

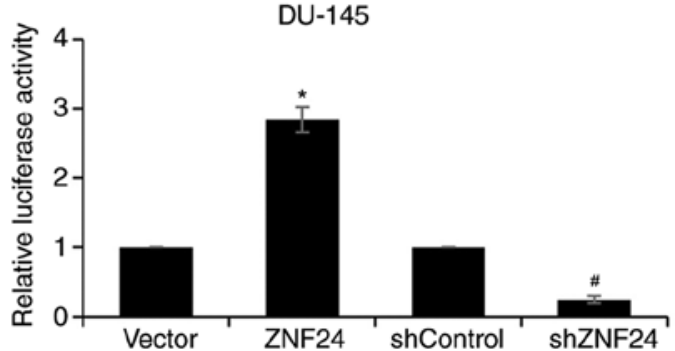

Figure 4. Twist1 is a target gene of ZNF24. (A) ChIP and quantitative ChIP assays were performed in order to determine whether ZNF24 could directly bind the promoter region of Twist1. (B) PC-3 and DU-145 cells were co-transfected with ZNF24 or shZNF24 and pGL3-Twist1 and Renilla luciferase reporter vector for $24 \mathrm{~h}$, followed by a dual luciferase assay to evaluate the relative luciferase activities. ${ }^{\text {"P }}<0.05$, ZNF24 vs. vector; ${ }^{\text {}} \mathrm{P}<0.05$, shZNF24 vs. shControl. ChIP, chromatin immunoprecipitation; IgG, immunoglobulin G; sh, short hairpin RNA; ZNF24, zinc finger protein 24.
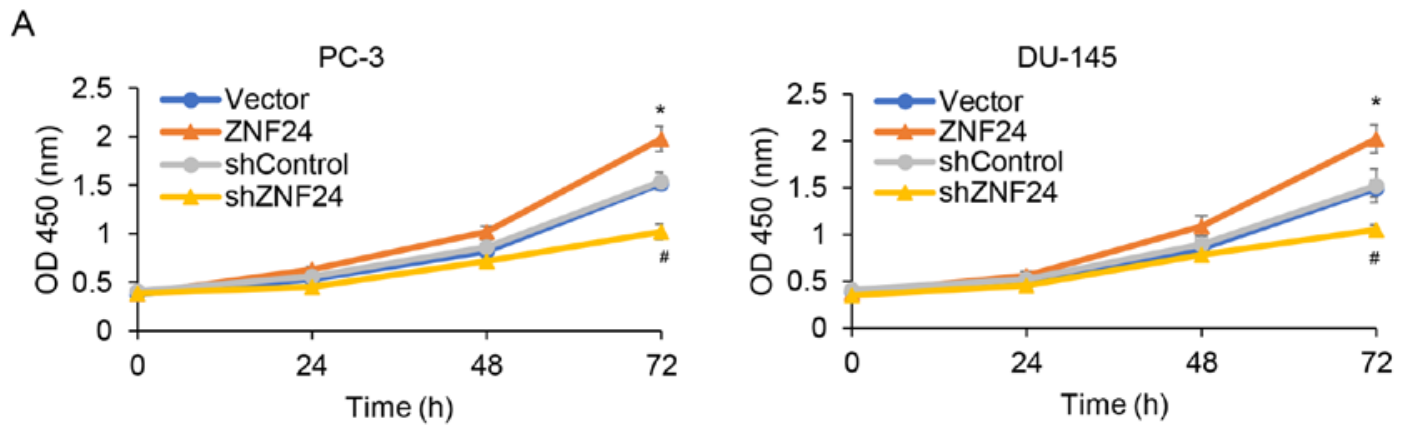

B
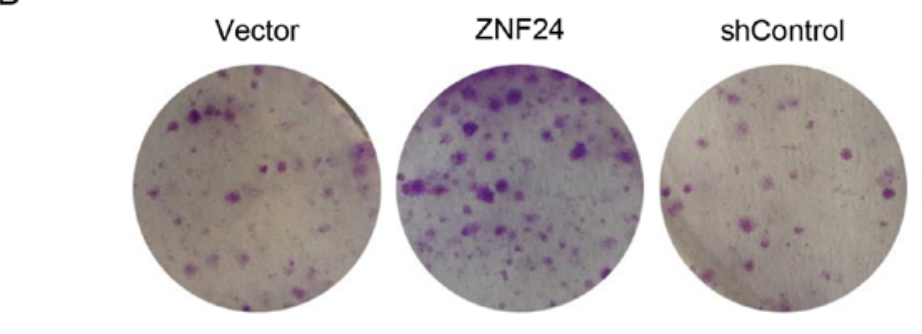

shZNF24

PC-3
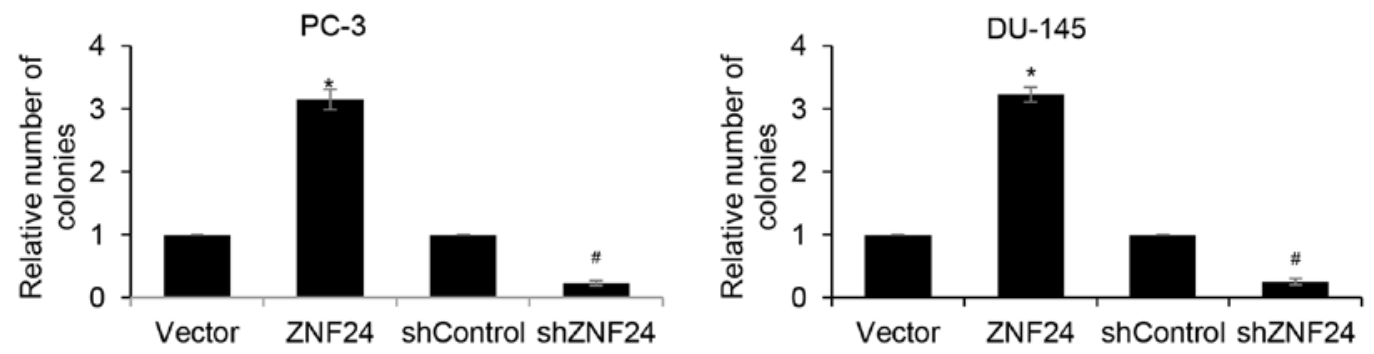

Figure 5. Upregulation of ZNF24 promotes prostate cancer cell proliferation. (A) ZNF24 was overexpressed or knocked-down in PC-3 and DU-145 cells. Cell Counting Kit- 8 assay was performed to detect the effect of ZNF24 on cell proliferation. ${ }^{~} \mathrm{P}<0.05$, ZNF24 vs. vector; ${ }^{~} \mathrm{P}<0.05$, shZNF24 vs. shControl. (B) ZNF24 was overexpressed or knocked down in PC-3 and DU-145 cells. Colony formation assays were performed to evaluate cancer cell growth. "P<0.05, ZNF24 vs. vector; " $\mathrm{P}<0.05$, shZNF24 vs. shControl. OD, optical density; sh, short hairpin RNA; ZNF24, zinc finger protein 24. 
when ZNF24 was overexpressed compared with the vector group $(\mathrm{P}<0.05$; Fig. 5A). Conversely, ZNF24 knockdown decreased the cell proliferation rate $(\mathrm{P}<0.05$; Fig. $5 \mathrm{~A})$. For the DU-145 cells, the change of proliferation rate at 24, 48 and $72 \mathrm{~h}$ after culture was determined to be in conformity with the PC-3 cells $(\mathrm{P}<0.05$; Fig. 5A). Subsequently, colony formation assays confirmed that ectopic expression of ZNF24 promoted cell proliferation and inhibition of ZNF24 suppressed cell proliferation in PC-3 and DU-145 cells ( $\mathrm{P}<0.05$; Fig. 5B). These results indicated that ZNF24 promoted $\mathrm{PCa}$ cell proliferation.

\section{Discussion}

ZNF24, also known as ZNF191 and KOX17 (7,31), contains four zinc finger motifs that encode putative DNA binding domains. In previous years, multiple studies have revealed that ZNF24 is involved in embryonic development $(10,13,14)$ and hematopoiesis (32), and negatively regulates vascular endothelial growth factor (VEGF) expression (33). In hepatocellular carcinoma, ZNF24 directly binding to the $\beta$-catenin promoter promotes cell proliferation (14). Liu et al (15) reported that microRNA-940 promotes tumor cell invasion and metastasis by downregulating ZNF24 in gastric cancer (GC), suggesting that ZNF24 serves as a tumor suppressor in GC. The contradicting results of previous studies indicate that ZNF24 plays different roles in different organizations. However, to the best of our knowledge, the detailed function of ZNF24 in PCa remains unknown.

The present study demonstrated that ZNF24 was frequently overexpressed in human $\mathrm{PCa}$ tissues and cell lines. Furthermore, the results revealed that overexpression of ZNF24 facilitated PCa cell migration and invasion in vitro through promoting EMT. Additionally, ZNF24 could transcriptionally regulate Twist1, a key transcription factor of the EMT process. The present study revealed that upregulation of ZNF24 increased Twist1 expression and inhibition of ZNF24 decreased Twist1 expression in PC-3 and DU-145 cells. Taken together, the data revealed that ZNF24 served as a novel oncogene in PCa and acted as a potential therapeutic target. However, a previous study indicated that ZNF24 functions as a negative regulator of developmental and tumor angiogenesis by directly binding to an 11-bp fragment of the VEGF proximal promoter and thus inhibiting VEGF transcription (33). Additionally, ZNF24 serves as a potential tumor suppressor and is inversely associated with microRNA-940 expression in GC (15). The opposing functions of ZNF24 in PCa and GC may be due to ZNF24 interacting with different proteins in different types of cancer.

Upregulation of ZNF24 was associated with tumor volume. Additionally, the present study revealed that ectopic expression or inhibition of ZNF24 could promote or suppress cell growth of the human PCa cell lines PC-3 and DU-145 in vitro. These results demonstrated that ZNF24 may be associated with cell proliferation of human $\mathrm{PCa}$. Consistent with previous studies, ZNF24 may serve a key role in cell proliferation during embryonic development $(10,13,14)$.

However, there were several limitations to the present study. First, it is preferable to perform immunohistochemistry assays to determine the expression of ZNF24 in PCa tissues. Furthermore, the effect of ZNF24 in vivo requires further investigation. In addition, the upstream genes of ZNF24 in PCa remain unknown. It would be useful to investigate whether the stages of PCa can also influence ZNF24 expression. There may be multiple proteins that up- or downregulate ZNF24 expression during different stages of PCa. An assay for RNA-sequencing and transposase-accessible chromatin using sequencing may be performed using diverse stages of PCa tissue samples, and potential transcription factors could be predicted. This may further decipher PCa development.

In summary, the present study demonstrated that ZNF24 served as an oncogene in PCa. Mechanistically,ZNF24 directly regulated Twist 1 expression to promote the EMT process, and contributed to the promotion of cell migration and invasion ability of PCa cells. In addition, ZNF24 also promoted cell proliferation. Therefore, the present study provided a strong rationale for ZNF24 as a therapeutic target in $\mathrm{PCa}$.

\section{Acknowledgements}

Not applicable.

\section{Funding}

No funding was received.

\section{Availability of data and materials}

The datasets used and/or analyzed during the current study are available from the corresponding author on reasonable request.

\section{Authors' contributions}

$\mathrm{XH}$ and NL conceived and designed the study. NL and XX performed the experiments. NL and XX wrote the paper. XH, NL and XX reviewed the manuscript. All authors read and approved the manuscript, and agreed to be accountable for all aspects of the research in ensuring that the accuracy or integrity of any part of the work are appropriately investigated and resolved.

\section{Ethics approval and consent to participate}

The Ethics Committee of Shenzhen People's Hospital approved the present study (approval no. LA2019013). All patients provided written informed consent.

\section{Patient consent for publication}

Not applicable.

\section{Competing interests}

The authors declare that they have no competing interests.

\section{References}

1. Siegel R, Naishadham D and Jemal A: Cancer statistics, 2013. CA Cancer J Clin 63: 11-30, 2013.

2. Panebianco V, Sciarra A, Marcantonio A, Forte V, Biondi T, Laghi A and Catalano C: Conventional imaging and multiparametric magnetic resonance (MRI, MRS, DWI, MRP) in the diagnosis of prostate cancer. Q J Nucl Med Mol Imaging 56: 331-342, 2012. 
3. Cusan L: Prostate cancer screening with PSA, DRE and TRUS Can J Oncol 4 (Suppl 1): S63-S64, 1994.

4. Crowell PD and Goldstein AS: Functional evidence that progenitor cells near sites of inflammation are precursors for aggressive prostate cancer. Mol Cell Oncol 4: e1279723, 2017.

5. Shafique K, Proctor MJ, McMillan DC, Qureshi K, Leung H and Morrison DS: Systemic inflammation and survival of patients with prostate cancer: Evidence from the glasgow inflammation outcome study. Prostate Cancer Prostatic Dis 15: 195-201, 2012.

6. Sfanos KS and De Marzo AM: Prostate cancer and inflammation: The evidence. Histopathology 60: 199-215, 2012

7. Han ZG, Zhang QH, Ye M, Kan LX, Gu BW, He KL, Shi SL, Zhou J, Fu G, Mao M, et al: Molecular cloning of six novel Krüppel-like zinc finger genes from hematopoietic cells and identification of a novel transregulatory domain KRNB. J Biol Chem 274: 35741-35748, 1999.

8. Edelstein LC and Collins T: The SCAN domain family of zinc finger transcription factors. Gene 359: 1-17, 2005.

9. Wang H, Sun R, Liu G, Yao M, Fei J and Shen H: Characterization of the target DNA sequence for the DNA-binding domain of zinc finger protein 191. Acta Biochim Biophys Sin (Shanghai) 40 704-710, 2008

10. Khalfallah O, Faucon-Biguet N, Nardelli J, Meloni R and Mallet J: Expression of the transcription factor Zfp191 during embryonic development in the mouse. Gene Expr Patterns 8: 148-154, 2008.

11. Prost JF, Nègre D, Cornet-Javaux F, Cortay JC, Cozzone AJ, Herbage D and Mallein-Gerin F: Isolation, cloning, and expression of a new murine zinc finger encoding gene. Biochim Biophys Acta 1447: 278-283, 1999.

12. Howng SY, Avila RL, Emery B, Traka M, Lin W, Watkins T, Cook S, Bronson R, Davisson M, Barres BA and Popko B: ZFP191 is required by oligodendrocytes for CNS myelination. Genes Dev 24: 301-311, 2010.

13. Li J, Chen X, Yang H, Wang S, Guo B, Yu L, Wang Z and Fu J: The zinc finger transcription factor 191 is required for early embryonic development and cell proliferation. Exp Cell Res 312: 3990-3998, 2006.

14. Liu G, Jiang S, Wang C, Jiang W, Liu Z, Liu C, Saiyin H, Yang $\mathrm{X}$, Shen S, Jiang D, et al: Zinc finger transcription factor 191 , directly binding to $\beta$-catenin promoter, promotes cell proliferation of hepatocellular carcinoma. Hepatology 55 1830-1839, 2012.

15. Liu X, Ge X, Zhang Z, Zhang X, Chang J, Wu Z, Tang W, Gan L, Sun M and Li J: MicroRNA-940 promotes tumor cell invasion and metastasis by downregulating ZNF24 in gastric cancer. Oncotarget 6: 25418-25428, 2015.

16. Khalfallah O, Ravassard P, Lagache CS, Fligny C, Serre A, Bayard E, Faucon-Biguet N, Mallet J, Meloni R and Nardelli J: Zinc finger protein 191 (ZNF191/Zfp191) is necessary to maintain neural cells as cycling progenitors. Stem Cells 27: 1643-1653, 2009.

17. Lv L, Zhang J, Wang P, Meng Q, Liang W and Zhang L: Zinc finger protein 191 deficiency attenuates vascular smooth muscle cell proliferation, migration, and intimal hyperplasia after endovascular arterial injury. J Vasc Surg 59: 500-509, 2014.

18. Thiery JP, Acloque H, Huang RY and Nieto MA: Epithelialmesenchymal transitions in development and disease. Cell 139 871-890, 2009.
19. Kalluri R and Weinberg RA: The basics of epithelial-mesenchymal transition. J Clin Invest 119: 1420-1428, 2009.

20. Thiery JP: Epithelial-mesenchymal transitions in development and pathologies. Curr Opin Cell Biol 15: 740-746, 2003.

21. Yao D, Dai C and Peng S: Mechanism of the mesenchymal-epithelial transition and its relationship with metastatic tumor formation. Mol Cancer Res 9: 1608-1620, 2011.

22. Wang X, Ling MT, Guan XY, Tsao SW, Cheung HW, Lee DT and Wong YC: Identification of a novel function of TWIST, a bHLH protein, in the development of acquired taxol resistance in human cancer cells. Oncogene 23: 474-482, 2004.

23. Yang $\mathrm{J}$ and Weinberg RA: Epithelial-mesenchymal transition: At the crossroads of development and tumor metastasis. Dev Cell 14: 818-829, 2008.

24. Epstein JI, Egevad L, Amin MB, Delahunt B, Srigley JR and Humphrey PA; Grading Committee: The 2014 international society of urological pathology (ISUP) consensus conference on gleason grading of prostatic carcinoma: Definition of grading patterns and proposal for a new grading system. Am J Surg Pathol 40: 244-252, 2016.

25. Buyyounouski MK, Choyke PL, McKenney JK, Sartor O, Sandler HM, Amin MB, Kattan MW, Lin DW: Prostate cancer-major changes in the American joint committee on cancer eighth edition cancer staging manual. CA Cancer J Clin 67: 245-253, 2017.

26. Livak KJ and Schmittgen TD: Analysis of relative gene expression data using real-time quantitative PCR and the 2(-Delta Delta $\mathrm{C}(\mathrm{T})$ ) method. Methods 25: 402-408, 2001

27. Roy R, Rodig S, Bielenberg D, Zurakowski D and Moses MA: ADAM12 transmembrane and secreted isoforms promote breast tumor growth: A distinct role for ADAM12-S protein in tumor metastasis. J Biol Chem 286: 20758-20768, 2011.

28. Yang J, Bielenberg DR, Rodig SJ, Doiron R, Clifton MC, Kung AL, Strong RK, Zurakowski D and Moses MA: Lipocalin 2 promotes breast cancer progression. Proc Natl Acad Sci USA 106: 3913-3918, 2009

29. Lamouille S, Xu J and Derynck R: Molecular mechanisms of epithelial-mesenchymal transition. Nat Rev Mol Cell Biol 15: 178-196, 2014.

30. Kumar A, Coleman I, Morrissey C, Zhang X, True LD, Gulati R, Etzioni R, Bolouri H, Montgomery B, White T, et al: Substantial interindividual and limited intraindividual genomic diversity among tumors from men with metastatic prostate cancer. Nat Med 22: 369-378, 2016.

31. Rousseau-Merck MF, Huebner K, Berger R and Thiesen HJ: Chromosomal localization of two human zinc finger protein genes, ZNF24 (KOX17) and ZNF29 (KOX26), to 18q12 and 17p13-p12, respectively. Genomics 9: 154-161, 1991.

32. Noll L, Peterson FC, Hayes PL, Volkman BF and Sander T: Heterodimer formation of the myeloid zinc finger 1 SCAN domain and association with promyelocytic leukemia nuclear bodies. Leuk Res 32: 1582-1592, 2008.

33. Harper J, Yan L, Loureiro RM, Wu I, Fang J, D'Amore PA and Moses MA: Repression of vascular endothelial growth factor expression by the zinc finger transcription factor ZNF24. Cancer Res 67: 8736-8741, 2007. 